\section{Causas múltiplas de morte por doenças crônico-degenerativas: uma análise multidimensional}

\author{
Multiple causes of death due \\ to non-communicable diseases: \\ a multidimensional analysis
}

\author{
${ }^{1}$ Escola de Enfermagem, \\ Universidade Federal \\ de Minas Gerais, \\ Belo Horizonte, Brasil. \\ 2 Escola de Veterinária, \\ Universidade Federal \\ de Minas Gerais, \\ Belo Horizonte, Brasil. \\ 3 Gerência de Regulação, \\ Epidemiologia \\ e Informação/Gerência \\ de Saúde Centro-Sul. \\ Secretaria Municipal \\ de Saúde de Belo Horizonte, \\ Belo Horizonte, Brasil. \\ Correspondência \\ E. M. Rezende \\ Rua Costa Sena 93/301, \\ Belo Horizonte, $M G$ \\ 30720-350, Brasil. \\ ednarez@enf.ufmg.br
}

\section{Abstract}

This study approaches multiple causes of death to analyze the associations between causes of death from non-communicable diseases, age, and gender in 3,106 death certificates issued in 1998, comprising individuals who were 20 years old or over and resided in Belo Horizonte, Minas Gerais State, Brazil. The multiple cause of death approach, by considering all the diagnoses mentioned in the death certificate, highlights conditions that are underestimated by the underlying-cause approach that has traditionally been used in mortality statistics. Association of causes of death was analyzed using the multidimensional data analysis method with the multiple correspondence factor analysis technique. The results reinforce the usefulness of the multiple causes approach to improve information on mortality and demonstrate important associations, such as hypertension and cerebrovascular diseases, obesity and diabetes or ischemic heart diseases, allowing to plan, prioritize, and reevaluate health actions.

Chronic Diseases; Mortality; Death Certificates
Edna Maria Rezende 1

Ivan Barbosa Machado Sampaio 2

Lenice Harumi Ishitani 3

Introdução

As declarações de óbito constituem uma fonte rica de informações extremamente útil para subsidiar investigações epidemiológicas visando à prevenção e à cura de doenças 1 . De acordo com a Organização Mundial da Saúde (OMS) 2 e também na opinião de Israel et al. 3 , Laurenti 4 e Santo 5, as estatísticas de mortalidade constituem instrumento fundamental nas áreas de administração e planejamento em saúde, epidemiologia e demografia.

Entre as variáveis usuais em estudos de mortalidade, a causa de morte vem se destacando como uma das mais importantes, especialmente, quando analisada sob as óticas do sexo e idade. Por outro lado, uma das grandes limitações das estatísticas oficiais de mortalidade é a sua restrição à tabulação de uma só causa - a causa básica de morte - como é então denominada. Esse procedimento implica em grande perda de informações especialmente quando muitos diagnósticos são mencionados no atestado de óbito. Chamblee \& Evans 6 ressaltam que, embora a seleção da causa básica de morte seja feita através de regras uniformes e padronizadas, são muitas vezes arbitrárias e nem sempre levam a uma seleção satisfatória. Assim, muitas doenças e lesões mencionadas na declaração de óbito não podem ser selecionadas como causa básica por determinismo das regras de seleção e, por isso, não aparecem nas 
estatísticas de mortalidade. O enfoque de causa básica era apropriado aos padrões de mortalidade do início do século passado quando poucos diagnósticos eram informados na declaração de óbito, já que as mortes eram devidas, principalmente, a doenças infecciosas agudas e a violência 3 .

As profundas transformações na estrutura populacional, decorrentes de quedas bruscas de mortalidade e fecundidade, deslocaram-se gradativamente dos grupos jovens aos mais idosos, modificando a incidência e a prevalência de doenças, bem como as principais causas de morte. Desse modo, doenças que acometiam mais a população infantil, como as infecciosas e parasitárias, vão perdendo importância em prol de outras, como as crônico-degenerativas mais incidentes na população adulta e idosa. Essas doenças, em geral, de longa duração vão se acumulando nos indivíduos, considerando o aumento relativo da proporção de idosos e a tendência crescente da expectativa de vida 7 . Assim, ao ocorrer, a morte será então determinada por diversas causas, tornando difícil a escolha de apenas uma para descrever processo tão complexo. Nesse caso, o modelo de causa básica torna-se insuficiente para explicar o perfil de morbimortalidade e subsidiar o planejamento de ações de prevenção.

O estudo de todos os diagnósticos mencionados na declaração de óbito (causas múltiplas) é o mais apropriado por considerar a morte o resultado da interação de diversas causas. Pela análise de causas múltiplas, pode-se dimensionar a magnitude real de diversas doenças que, muitas vezes, permaneciam ocultas devido ao enfoque de causa básica e às imposições das regras de seleção 3,4,6,8,9. Para Machado 10, a tabulação de causas múltiplas permite, ainda, explorar a heterogeneidade populacional, discriminar aglomerados de causas e delinear perfis de morbimortalidade. Embora reconhecida a importância das causas múltiplas de morte para aprimoramento das estatísticas de mortalidade, seu uso é ainda restrito, considerando as dificuldades para operacionalização, como o grande volume de dados a ser processado, não obstante o uso do computador ter facilitado em muito esse processo 11. Além disso, não estão internacionalmente padronizados e incorporados à Classificação Estatística Internacional de Doenças e Problemas Relacionados à Saúde (CID) a conceituação e os procedimentos para codificação e tabulação das causas múltiplas de morte, o que dificulta o processamento e futuras comparações, considerando o número crescente de países, instituições e pesquisadores que estão fazendo uso dessa metodologia 4,5 .
Na opinião de Laurenti \& Buchalla 12, para a elaboração de estatísticas de causas múltiplas, não basta simplesmente codificar todos os diagnósticos informados. São necessárias regras, normas e orientações que considerem os interesses clínico e epidemiológico. Olson et al. 13 ressaltam ainda a dificuldade de determinar a significância estatística em associação de causas de morte, já que os métodos utilizados partem do pressuposto de independência das variáveis, portanto, não aplicáveis ao enfoque de causas múltiplas, cuja premissa é a interação entre as causas.

Judez 14 e Sampaio (Sampaio IBM. Relatório das atividades de pós-doutorado desenvolvidas no período de 03/Set/92 a 04/Mar/ 93. Relatório de pós-doutorado apresentado ao Grupo de Estatística, Universidade Politécnica de Madrid) sugerem, para estudo de associações entre variáveis, o método de Análise de Dados Multidimensionais. As principais vantagens, nesse caso, são a aplicabilidade a grande contingente de dados e a não utilização de testes inferenciais. Citando as afirmações de Benzecri et al., Sampaio ressalta que, sob essa concepção, o modelo é que deve seguir os dados, e as flagrantes soluções gráficas deixam os testes estatísticos e sua validade em segundo plano. Essa metodologia, empregada por Volpe $15 \mathrm{e}$ por Trópia 16, parece também instrumento de grande valia no estudo das associações de causas de morte.

Pretendeu-se, neste trabalho, estabelecer, para as mortes por doenças crônico-degenerativas, as relações sob os enfoques de causa básica e causas múltiplas e identificar, através da análise multidimensional e sob a abordagem de causas múltiplas, as interações e associações entre causas de morte, sexo e idade.

\section{Material e métodos}

O estudo foi baseado nas informações dos óbitos ocorridos em 1998, em pessoas que residiam em Belo Horizonte, Minas Gerais, Brasil. O banco de dados foi disponibilizado pelo Centro de Estatística e Informação da Fundação João Pinheiro, responsável pela codificação e processamento das declarações de óbito até 1998. Escolheu-se o referido ano por ser o último em que as declarações de óbito foram codificadas e processadas pela referida Fundação, submetidas, portanto, a uma mesma equipe de codificadores. A codificação das causas de morte foi feita com base na décima revisão da CID (CID-10) 17. Para seleção da causa básica, utilizou-se o Sistema de Seleção de Causa Básica 
(SCB-10), programa proposto por Santo \& Pinheiro 18.

Selecionaram-se, para revisão, as declarações de óbito cuja causa básica, representada por um único código da CID-10, indicava a combinação de duas ou mais causas. Essa seleção foi feita para todas as causas mencionadas na Tabela 1 - "Sumário das Associações pelo Número de Código", volume 2 da CID-10 19. Tal procedimento foi necessário já que a CID, estruturada para atender ao princípio da causa básica de morte, dispõe de categorias para classificar duas ou mais afecções conjuntamente. Nesse caso, como não existem ainda padronizações para codificação das causas múltiplas, os diagnósticos combinados foram desmembrados, uma vez que se pretendia captar, para o estudo, todas as causas como foram mencionadas na declaração de óbito original. Um exemplo, nesse caso, seria a doença cardíaca e renal hipertensiva, combinada pela ocorrência da dilatação cardíaca, esclerose renal e hipertensão. Avaliaram-se apenas as mortes naturais, assim definidas quando a causa básica era representada pelos códigos das seções de "A" a "R" da CID-10 17,19.

As variáveis selecionadas para este estudo foram sexo, idade e causas de morte. Para a variável sexo, foram estabelecidas as categorias: masculino e feminino. Para a idade, definiramse quatro categorias, a saber: ID1 para os indivíduos de 20 a 39 anos; ID2 para 40 a 59 anos; ID3 para 60 a 79 anos e ID4 para os indivíduos com 80 anos e mais. As causas de morte foram analisadas sob os enfoques de causa básica e causas múltiplas, e o estudo das associações, somente em relação às últimas. A causa básica foi definida conforme preconizado pela OMS, como a doença ou lesão que iniciou a cadeia de eventos patológicos e que levou diretamente à morte 19 . As causas múltiplas foram entendidas como o conjunto de todas as causas mencionadas na declaração de óbito, sem distinção de classificação em básica, conseqüenciais e contribuintes, conforme proposto por Laurenti 4 e Santo 5.

Os diagnósticos mencionados na declaração de óbito foram agrupados conforme a lista de tabulação da CID-10 e com base, também, na freqüência dos diagnósticos e interesse dos pesquisadores quanto à relevância das causas. Assim optou-se por um nível de agregação de causas maior do que aquele em que os dados foram produzidos, garantindo-se, entretanto, que todas as duplicações e multiplicações de diagnósticos fossem eliminadas, conforme sugerido por Santo 5 . Desse modo, quando determinadas afecções mencionadas em única declaração pertenciam ao mesmo agrupamento, só ele era considerado. Foi o caso, por exemplo, de aparecer, em um mesmo atestado de óbito, infarto agudo do miocárdio (I21.9), insuficiência coronariana (I24.8) e doença aterosclerótica do coração (I25.1). Como pertenciam ao mesmo agrupamento, doenças isquêmicas do coração (I20-I25), não seriam contadas individualmente. Seguindo as recomendações do Manual of Mortality Analysis 2, de Santo 5 e de Olson et al. 13, selecionaram-se, para serem analisados, grupos específicos de causas de morte, classificados e codificados como especificado a seguir: diabetes mellitus (DM) - E10 a E14; obesidade (OB) - E66; doenças hipertensivas (DH) - I10 a I13; doenças isquêmicas do coração (DI) - I20 a I25; doenças cerebrovasculares (DC) - I60 a I69; aterosclerose (AT) - I70 e insuficiência renal (IR) - N17 a N19. Para cada grupo de causas, duas categorias foram criadas indicando a presença da enfermidade (S) ou a sua ausência (N). Assim, cada variável causa ficou representada pela letra do grupo, seguida de "S" (DMS, OBS etc.) ou "N" (DMN, OBN etc.). Para representação gráfica, apenas as variáveis que indicavam a presença da condição foram representadas. As causas de morte foram analisadas através das respectivas freqüências, determinando-se a razão entre causas múltiplas e básica. Para o estudo das associações, foram eliminadas as observações (declarações de óbito) em que apenas uma causa foi mencionada e também aquelas que correspondiam a óbitos em que o sexo e a idade eram ignorados, já que cada observação teria que estar presente em uma categoria de cada variável. Foram eliminadas 2.785 declarações de óbito por terem menção de apenas uma causa; 26 , pela não definição de sexo, e duas, por falta de idade.

O método utilizado para estudo das associações de causas de morte foi o de Análise de Dados Multidimensionais (Análise Multivariada) por meio da técnica de Análise Fatorial de Correspondência Múltipla. Desenvolvida através da elaboração de um quadro binário, a técnica emprega conceitos basicamente qualitativos, em que cada variável possui categorias distintas e exclusivas. Cada categoria recebe o valor " 1 " se a condição analisada estiver presente e "0" se o caráter for ausente 14 (Sampaio IBM. Relatório das atividades de pós-doutorado desenvolvidas no período de 03/Set/92 a 04/Mar/93. Relatório de pós-doutorado apresentado ao Grupo de Estatística, Universidade Politécnica de Madrid). Após formatação, os dados foram processados pelo programa SAS 20, e os resultados representados graficamente, sem testes inferenciais. A representação gráfica das variáveis exige o cálculo dos eixos fatoriais, 
das coordenadas dessas sobre os referidos eixos e do percentual da inércia explicada. Cada eixo é uma combinação linear de todas as variáveis estudadas. Se existirem " $\mathrm{P}$ " variáveis, $\mathrm{o}$ sistema consegue se justificar plenamente com "P" eixos. Entretanto, para a apreciação do fenômeno, utiliza-se uma dimensão perceptível (até três eixos), esperando-se que a inércia então justificada seja alta (em torno de 70,0\%). A interpretação é baseada na localização dos pontos, e estão associados aqueles encontrados na mesma direção em relação à origem e na mesma região do espaço - quadrante 20 . A distância entre eles será avaliada relativamente. Variáveis mais afastadas dos eixos contribuem mais para a variação. Variáveis próximas, em mesmo quadrante, usufruem das mesmas condições observadas, e variáveis em quadrantes opostos serão antagônicas.

\section{Resultados e discussão}

Em 1998, foram registrados 13.999 óbitos na população residente em Belo Horizonte, estimada, pela Secretaria Municipal de Saúde, para aquele ano, em 2.159.320 habitantes. Dessas mortes, 12.359 foram por causas naturais, totalizando 31.289 menções, depois de eliminadas as multiplicações e duplicações de diagnósticos.

A sobremortalidade masculina foi confirmada, neste estudo, com um percentual de 51,4\%. Cerca de $57,4 \%$ dos óbitos concentraram-se em idades acima de sessenta anos correspondente a aproximadamente $8,7 \%$ da população total do município. Os atestados de óbito tinham em média de 2,6 diagnósticos. Aqueles com, no máximo, três diagnósticos representaram $80,4 \%$, e não chegou a 1,0\% o número de declarações com mais de cinco diagnósticos, conforme evidenciado na Tabela 1.

Os atestados com menção de apenas uma causa somaram 2.785 (22,5\%), de modo que, em $77,5 \%$ deles, havia mais de uma afecção informada. Esse percentual retrata o montante de declarações em que ocorreria perda de informações, se analisadas sob a perspectiva de causa básica. Para Israel et al. 3 e Santo \& Pinheiro 11, a análise do número de diagnósticos mencionados na declaração de óbito é de fundamental importância já que, além de outros aspectos, poderá revelar tanto a qualidade da assistência médica recebida como a qualidade do preenchimento da declaração. Janssen 8 ressalta que o número de diagnósticos por atestado de óbito pode refletir ainda vários fatores como o tipo da doença envolvida, características da população, como cor branca ou negra, variação geográfica de residência rural ou urbana e capacidade instalada, como número de hospitais e outros serviços de saúde disponíveis. O valor médio do número de diagnóstico encontrado neste estudo, ligeiramente inferior ao encontrado por Santo 5 para o distrito de São Paulo em 1983 e ao descrito por Ishitani \& França ${ }^{21}$ para a região Centro-Sul de Belo Horizonte, poderia ser explicado pelos fatores acima mencionados.

O grupo de afecções enfocado neste trabalho, formado por DM, DH, DI, DC, AT, IR e OB, correspondeu a 3.106 óbitos $(25,0 \%$ do total), sob o enfoque de causa básica. Considerando o número de vezes em que essas doenças foram mencionadas na declaração de óbito, totalizaram 6.127 diagnósticos. Esse grupo de causas foi definido considerando que, com o aumento relativo da proporção de idosos decorrente da maior expectativa de vida, houve um crescimento da prevalência das doenças, especialmente as crônico-degenerativas e as relacionadas às formas de vida. Além disso, de acordo com o Manual of Mortality Analysis 2 e considerações de Santo 5 , conclusões significativas sobre associações de causas de morte só poderão ser obtidas se as comparações forem feitas entre grupos específicos, claramente definidos e cientificamente válidos. Um exemplo, nesse caso, seria a hipertensão arterial e as doenças cerebrovasculares, considerando a alta freqüência em que são juntamente mencionadas.

A Tabela 2 compara as causas de morte estudadas sob os enfoques de causa básica e causas múltiplas, estabelecendo-se a razão entre ambos. Observam-se altas razões, iguais ou maiores que cinco, para a aterosclerose, obesidade e insuficiência renal, geralmente consideradas como causas terminais ou que contribuem para a morte, pouco selecionadas como causa básica. Os incrementos encontrados em relação ao diabetes mellitus, sob a perspectiva de causas múltiplas, também foram descritos por Melo et al. 22 e por Franco et al. 23. O impacto das doenças hipertensivas, mencionadas mais de quatro vezes em relação à causa básica, reforça os resultados encontrados por Ishitani \& França 21 . Esses achados evidenciam a importância das causas múltiplas para se dimensionar a magnitude real de determinadas afecções, de forma especial, aquelas que demandam intervenções preventivas mais precoces, no sentido de evitar complicações mais sérias, além de gastos sociais e financeiros. Constituem, portanto, uma advertência para gestores e planejadores de serviços de saúde que utilizam as estatísticas oficiais de mortalidade como base para elaboração de políticas de preven- 
ção e controle. Em relação às doenças cerebrovasculares e às doenças isquêmicas do coração, que assumem importante papel no quadro de morbimortalidade atual, cabe ressaltar que a razão encontrada, próxima à unidade, é devida ao fato de ambas serem protegidas pelas regras de seleção da causa básica, especialmente em relação às doenças hipertensivas, que, nesse caso, são as afecções preteridas 5,19,21.

\section{Associação de causas de morte}

Para estudo das associações de causas de morte, refutou-se a abordagem clássica baseada na causa básica, de modo que as diversas afecções mencionadas na declaração de óbito foram analisadas conjuntamente como causas múltiplas, mas sem distinção se básicas, conseqüenciais ou contribuintes. Esse tipo de associação, denominado por Santo 5 como multidirecional, se faz independentemente da qualificação das causas de morte ou de suas posições no atestado de óbito. Pelas limitações de processamento, acrescenta ainda o autor, essa análise não foi por ele utilizada. Assim a técnica usada no presente trabalho, Análise Fatorial de Correspondência Múltipla, difere daquela empregada por Santo \& Pinheiro 11 e Ishitani \& França ${ }^{21}$ para análise da associação das causas múltiplas de morte. Sua escolha, conforme aspectos já mencionados, foi devida a sua aplicabilidade a grande contingente de dados, a não utilização de testes inferenciais e por possibilitar fácil visualização gráfica das associações 14,20 (Sampaio IBM. Relatório das atividades de pósdoutorado desenvolvidas no período de 03 / Set/92 a 04/Mar/93. Relatório de pós-doutorado apresentado ao Grupo de Estatística, Universidade Politécnica de Madrid).

A Figura 1 evidencia a forte associação entre obesidade, doenças isquêmicas do coração e aterosclerose, especialmente na idade de 60 a 79 anos. A esse grupo associam-se ainda, embora com menos intensidade, as doenças hipertensivas e as cerebrovasculares, essas fortemente associadas entre si, especialmente em mulheres mais idosas, acima de oitenta anos, e em homens mais jovens, como mostra a Figura 2. Nesse caso, a inércia justificada nos três eixos foi $58,0 \%$, o que significa um bom domínio sobre a variação experimentada pelas variáveis. Outros estudos, como o de Santo 5 e o de Ishitani \& França 21, procuraram resgatar a importância e a inter-relação das doenças hipertensivas como fator de risco para doenças cerebrovasculares e doenças isquêmicas do coração, já que, pelas regras de seleção da causa básica, a doença hipertensiva é preterida em rela-
Tabela 1

Freqüências e percentuais, simples e acumulados, de diagnósticos por declaração de óbito. Mortes naturais. Belo Horizonte, Minas Gerais, Brasil, 1998.

\begin{tabular}{lrrrr}
\hline $\begin{array}{l}\text { Número de } \\
\text { diagnósticos }\end{array}$ & Freqüência & $\%$ & $\begin{array}{r}\text { Óbitos } \\
\text { Freqüência } \\
\text { acumulada }\end{array}$ & $\%$ acumulado \\
\hline 1 & 2.785 & 22,5 & 2.785 & 22,5 \\
2 & 3.544 & 28,7 & 6.329 & 51,2 \\
3 & 3.606 & 29,2 & 9.935 & 80,4 \\
4 & 1.658 & 13,4 & 11.593 & 93,8 \\
5 & 652 & 5,3 & 12.245 & 99,1 \\
6 e + & 114 & 0,9 & 12.359 & 100,0 \\
\hline
\end{tabular}

Freqüência de óbitos = número de declarações de óbito.

Tabela 2

Número de óbitos por causas de morte segundo total de menções, causa básica e razão. Mortes naturais. Belo Horizonte, Minas Gerais, Brasil, 1998.

\begin{tabular}{lccc}
\hline Causas de morte & $\begin{array}{c}\text { Total de } \\
\text { menções }(\mathbf{m})\end{array}$ & $\begin{array}{c}\text { Causa básica } \\
\text { (b) }\end{array}$ & $\begin{array}{r}\text { Razão } \\
\text { (m/b) }\end{array}$ \\
\hline Diabetes mellitus & 813 & 291 & 2,79 \\
Obesidade & 43 & 8 & 5,38 \\
Doenças hipertensivas & 1.309 & 296 & 4,42 \\
Doenças isquêmicas do coração & 1.332 & 1.083 & 1,23 \\
Doenças cerebrovasculares & 1.669 & 1.255 & 1,33 \\
Aterosclerose & 260 & 52 & 5,00 \\
Insuficiência renal & 701 & 121 & 5,79 \\
Total & 6.127 & 3.106 & 1,97 \\
\hline
\end{tabular}

Total de menções = causas múltiplas .

ção às últimas. Destacaram a associação recíproca entre doenças hipertensivas e cerebrovasculares, também observada neste trabalho, o que pressupõe que tal associação aumentaria o risco de morte por doença cerebrovascular, conforme encontrado por Currie et al. $24 \mathrm{e}$ Kuller et al. 25 .

A associação entre obesidade, doenças hipertensivas, diabetes mellitus e insuficiência renal, mais evidente entre 60 e 79 anos (Figura 3), reforça a influência da obesidade para o aparecimento tanto do diabetes quanto da doença hipertensiva. Essa última possivelmente também conseqüente ao diabetes e juntamente com ele pode implicar em sérias complicações, entre elas, a insuficiência renal. A explicação de 43,0\% da inércia, para as projeções dessas variáveis nos três primeiros eixos fatori- 
Figura 1

Representação gráfica das variáveis estudadas nos dois primeiros eixos da Análise Fatorial de Correspondência, no universo de mortes por doenças crônico-degenerativas, em indivíduos de vinte anos e mais. Belo Horizonte, Minas Gerais, Brasil, 1998.

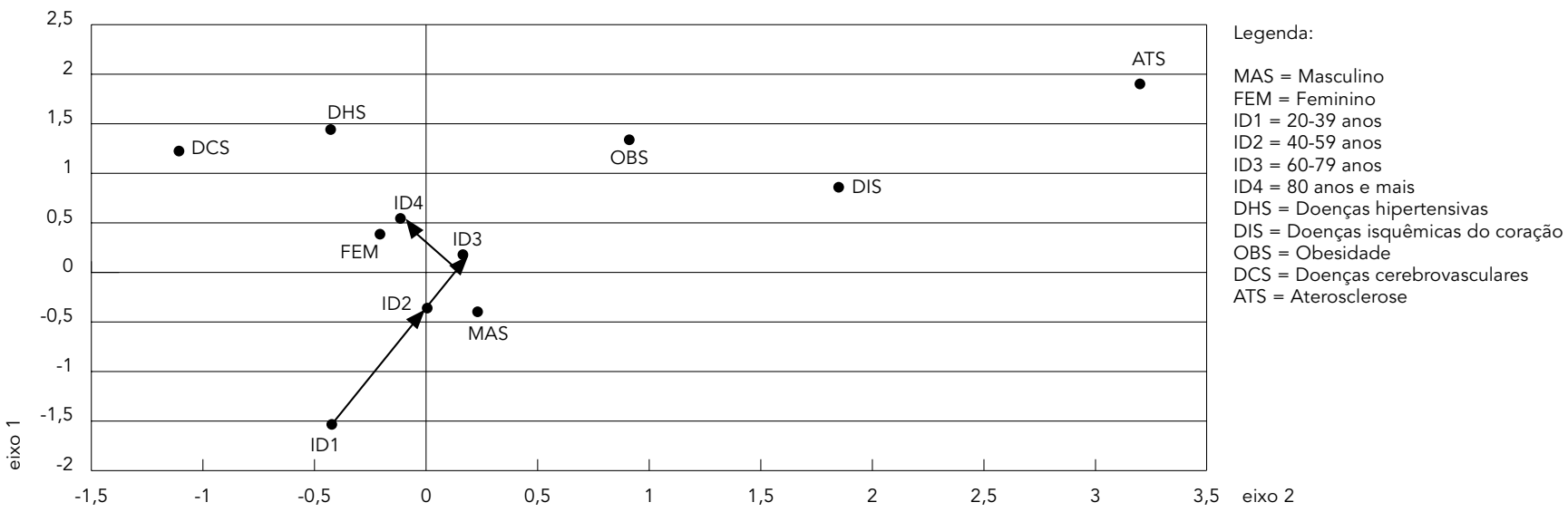

ais, não compromete as inter-relações encontradas, já que, segundo Sampaio (Sampaio IBM. Relatório das atividades de pós-doutorado desenvolvidas no período de 03/Set/92 a 04/Mar/ 93. Relatório de pós-doutorado apresentado ao Grupo de Estatística, Universidade Politécnica de Madrid), mesmo com justificativas de inércias mais baixas, inferiores a 70,0\%, possivelmente por instabilidade da resposta medida, ainda assim, as associações buscadas podem claramente se manifestar. A associação entre diabetes mellitus, doenças hipertensivas e complicações renais também foi evidenciada por Laurenti et al. 4,26 e Ishitani \& França 21.

A forte associação de obesidade tanto com doenças isquêmicas do coração (Figura 1) como com as doenças hipertensivas e diabetes mellitus (Figura 3) confirma as preocupações de Monteiro \& Conde 27. Com base em publicações da OMS, advertem sobre as conseqüências advindas da obesidade, que incluem desde dificuldades respiratórias até enfermidades parcialmente letais, entre elas, as doenças cardiovasculares e o diabetes não-insulino dependente. Nesse sentido, Olson et al. 13, há várias décadas, defendem a investigação do diabetes através de causas múltiplas, já que, pelo enfoque de causa básica, permaneceriam desconhecidos possíveis fatores causais, sua extensão e efeitos na mortalidade. $\mathrm{O}$ diabetes mellitus tem um impacto social importante pelos custos não somente com mortalidade prematura mas tam- bém com morbidade, resultando em gastos com a atenção médica, tratamento, incapacidades e dias perdidos de trabalho.

As diversas associações explicitadas neste estudo colocam em evidência informações importantes que, sob o enfoque de causa básica, permaneceriam obscuras. Cabe ressaltar, entretanto, que a análise das causas múltiplas de morte, segundo Israel et al. 3 , está na dependência da magnitude com que os diagnósticos são mencionados pelos médicos, na declaração de óbito. A exemplo do que acontece em relação à causa básica, as causas múltiplas de morte resultam da opinião médica e podem variar segundo a escola, área geográfica e compreensão sobre qual causa deverá ser incluída na declaração. Nesse caso, a própria exigência para a informação da causa básica desestimula a descrição completa do curso clínico da doença que levou à morte. Laurenti 4 ressalta a necessidade de instruir os médicos para o preenchimento correto da declaração no sentido de tirar o máximo proveito das informações e coloca que o uso exclusivo do atestado de óbito, como fonte única de dados, é outro fator limitante, já que informações complementares em prontuários, autópsias e entrevistas melhoram substancialmente as estatísticas. À luz desses conhecimentos, programas mais concretos poderão ser elaborados visando às ações de prevenção das doenças, bem como de suas complicações, particularmente neste momento em 
que a atenção básica vem sendo grandemente enfatizada. Nesse sentido, Lotufo \& Lolio 28 atribuem ao serviço público a responsabilidade de coordenar os esforços da sociedade para reduzir a morbidade e mortalidade pelas doenças cardiovasculares de modo geral. Entre as medidas de controle, destacam o desestímulo ao tabagismo, o incentivo à adoção de hábitos dietéticos saudáveis e, particularmente, campanhas de massa para detecção precoce, tratamento e controle da hipertensão arterial.

\section{Considerações finais}

O alto percentual de declarações de óbito com mais de um diagnóstico, encontrado neste estudo, retrata a perda de informações caso as mortes fossem analisadas sob a perspectiva de causa básica e evidencia o impacto das causas múltiplas para melhorar as informações em mortalidade. A real magnitude das doenças hipertensivas, explicitadas sob o enfoque de causas múltiplas, e as diversas associações que a envolvem, entre elas, as doenças isquêmicas do coração e as cerebrovasculares, evidenciam a necessidade de programas de prevenção e controle que incluam, particularmente, a detecção precoce e o monitoramento da doença. As implicações vasculares e renais associadas ao diabetes mellitus suscitam a necessidade de investimentos em programas educativos, no sentido de prevenir a doença e evitar suas complicações. As conseqüências da obesidade constituem uma advertência para investimentos na qualidade de vida, especialmente, em relação à melhoria dos hábitos alimentares e ao combate ao sedentarismo. O método de Análise de Dados Multidimensionais e a técnica de Análise Fatorial de Correspondência Múltipla foram adequados para estabelecer as associações entre causas de morte, idade e sexo, de forma a subsidiar gestores e planejadores de saúde, em definições de políticas e prioridades. Medidas de saúde pública tais como ações educativas, tratamento, assistência e reabilitação social e profissional, voltadas para prevenção e monitoramento seriam, certamente, a melhor forma para reduzir a incidência das doenças crônicodegenerativas ou, pelo menos, retardar as suas conseqüências.
Figura 2

Representação gráfica das variáveis estudadas nos dois primeiros eixos da Análise Fatorial de Correspondência, no universo de mortes por doenças crônico-degenerativas, em indivíduos de vinte anos e mais.

Belo Horizonte, Minas Gerais, Brasil, 1998.

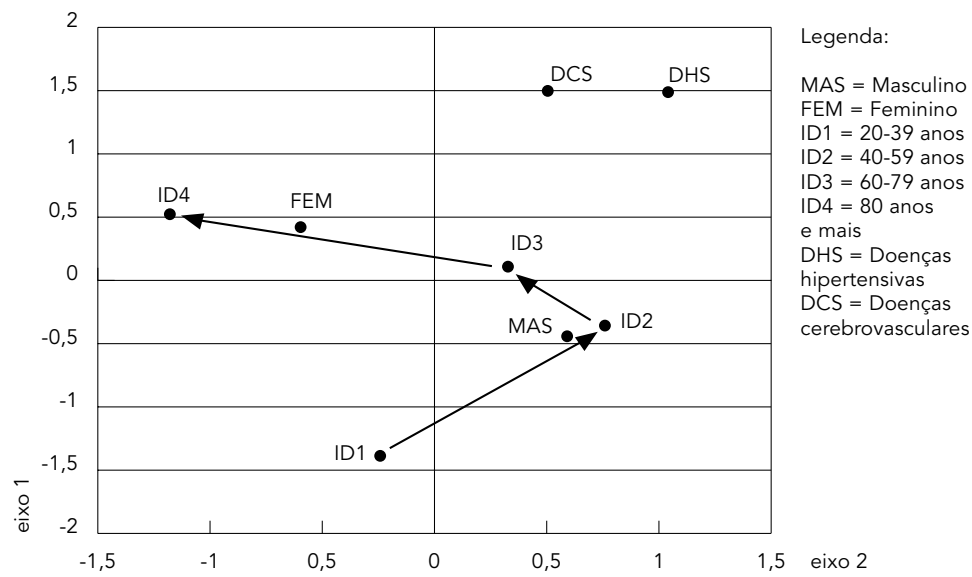

Figura 3

Representação gráfica das variáveis estudadas nos dois primeiros eixos da Análise Fatorial de Correspondência, no universo de mortes por doenças crônico-degenerativas, em indivíduos de vinte anos e mais.

Belo Horizonte, Minas Gerais, Brasil, 1998.

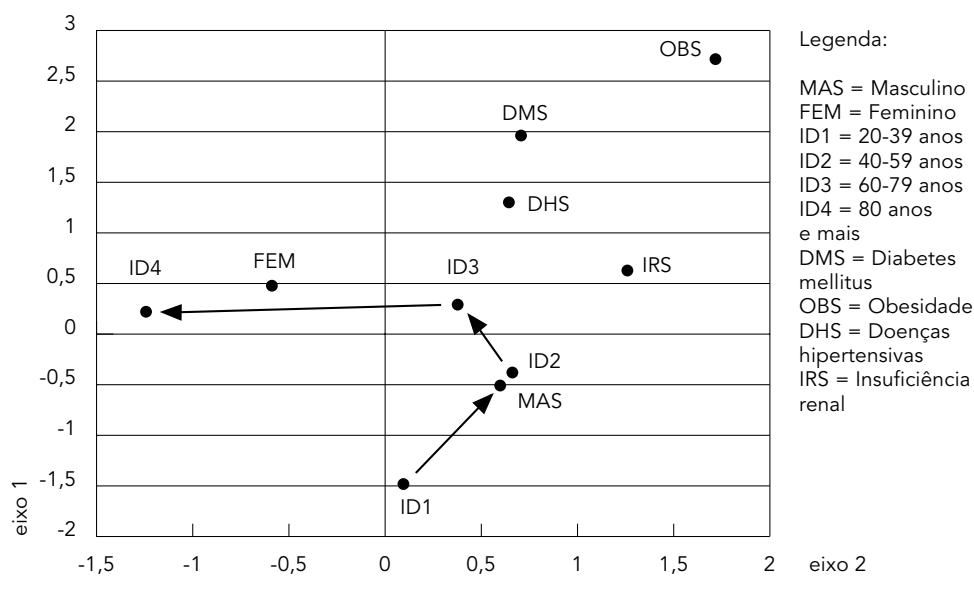




\section{Resumo}

Estudaram-se, sob o enfoque de causas múltiplas, as associações entre causas de morte por doenças crônico-degenerativas, idade e sexo, em 3.106 declarações de óbitos ocorridos em 1998, em pessoas com vinte anos e mais, que residiam em Belo Horizonte, Minas Gerais, Brasil. A análise das causas múltiplas, por considerar todos os diagnósticos da declaração de óbito, pode evidenciar afecções não selecionadas pelo enfoque de causa básica, tradicionalmente utilizado nas estatísticas de mortalidade. A associação de causas de morte foi analisada pelo método de Análise Multidimensional, através da técnica de Análise Fatorial de Correspondência Múltipla. Os resultados encontrados reforçam a utilidade das causas múltiplas de morte para melhorar as informações em mortalidade e evidenciam importantes associações tais como doenças hipertensivas e cerebrovasculares, obesidade e diabetes ou doenças isquêmicas do coração, informações essas que possibilitam planejar, priorizar e reavaliar as ações de saúde.

Doenças Crônicas; Mortalidade; Atestados de Óbito

\section{Colaboradores}

E. M. Rezende participou na elaboração do artigo, revisão da literatura e descrição da metodologia. I. B. M. Sampaio contribuiu na análise e discussão da aplicação do método de Análise Multidimensional de Dados e da técnica de Análise Fatorial de Correspondência Múltipla. L. H. Ishitani colaborou na análise e discussão das causas de morte, na utilização do enfoque de Causas Múltiplas, bem como na interpretação das associações de causas de morte evidenciadas. Todos os autores participaram da análise dos dados e discussão dos resultados relacionados ao método de Análise Multidimensional e ao enfoque de Causas Múltiplas.

\section{Referências}

1. Goodman RA, Manton KG, Nolan Jr. TF, Bregman DJ, Hinman AR. Mortality data analysis using a multiple-cause approach. JAMA 1982; 247:793-6.

2. World Health Organization. Dissemination on statistical information. Manual of mortlity analysis. A manual on methods of analysis of national mortality satitistics for public purposes. Geneva: World Health Organization; 1977.

3. Israel RA, Rosenberg HM, Curtin LR. Analytical potencial for multiple cause-of-death data. Am J Epidemiol 1986; 124:161-79.

4. Laurenti R. Causas múltiplas de morte [Tese de Livre Docência]. São Paulo: Faculdade de Saúde Pública, Universidade de São Paulo; 1973.

5. Santo AH. Causas múltiplas de morte: formas de apresentação e métodos de análise [Tese de Doutorado]. São Paulo: Universidade de São Paulo; 1988.

6. Chamblee RF, Evans MC. New dimensions in cause of death statistics. Am J Public Health 1982; 72:1265-70.

7. Barreto ML, Carmo EH. Mudanças em padrões de morbimortalidade: conceitos e métodos. In: Monteiro CA, organizador. Velhos e novos males da saúde no Brasil: evolução do país e suas doenças. São Paulo: Editora Hucitec/Núcleo de Pesquisas Epidemiológicas em Nutrição e Saúde, Universidade de São Paulo; 2000. p. 17-30.

8. Janssen TA. Importance of tabulating multiple causes of death. Am J Public Health 1940; 30:871-9.

9. Puffer RR. Estudio de multiplas causas de defunción. Bol Oficina Sanit Panam 1970; 69:93-114.

10. Machado CJ. Perfis de morbimortalidade infantil no Estado de São Paulo, 1994: uma aplicação de Grade of Membership à análise de causas múltiplas de morte [Dissertação de Mestrado]. Belo Horizonte: Faculdade de Ciências Econômicas, Universidade Federal de Minas Gerais; 1997.

11. Santo AH, Pinheiro CE. Tabulador de causas múltiplas de morte. Rev Bras Epidemiol 1999; 2:122-4.

12. Laurenti R, Buchalla CM. A elaboração de estatísticas de mortalidade segundo causas múltiplas. Rev Bras Epidemiol 2000; 3:21-8.

13. Olson FE, Norris FD, Hammes LM, Shipley PW. A study of multiple causes of death in California. J Chronic Dis 1962; 15:157-70.

14. Judez LA. Análisis factorial de correspondencias. In: Judez LA, editor. Técnicas de análisis de datos multidimensionales. Madrid: Ministerio de Agricultura, Pesca y Alimentación; 1989. p. 69-114.

15. Volpe FM. Eletroconvulsoterapia para episódios maníacos em um hospital de Belo Horizonte, 1996 a 2000 [Dissertação de Mestrado]. Belo Horizonte: Universidade Federal de Minas Gerais; 2002.

16. Trópia MAS. Fatores predisponentes para deficiência de vitamina $\mathrm{A}$ em escolares da área rural de novo cruzeiro - Minas Gerais - Brasil [Tese de Doutorado]. Belo Horizonte: Universidade Federal de Minas Gerais; 2002.

17. Organização Mundial da Saúde. Classificação Estatística Internacional de Doenças e Problemas Relacionados à Saúde - 10a Revisão. v. 1. São Paulo: Edusp; 1995.

18. Santo AH, Pinheiro CE. Uso do microcomputador 
na seleção de causa básica de morte. Bol Oficina Sanit Panam 1995; 119:319-26.

19. Organização Mundial da Saúde. Manual de instrução da Classificação Estatística Internacional de Doenças e Problemas Relacionados à Saúde 10ạ revisão. v. 2. São Paulo: Edusp; 1994.

20. Khattree R, Dayanand NN. Multivariate data reduction and discrimination with SAS software. Cary: SAS Institute Inc.; 2000.

21. Ishitani LH, França E. Doenças crônico-degenerativas em adultos da região Centro-Sul de Belo Horizonte: análise sob a perspectiva de causas múltiplas de morte. Inf Epidemiol SUS 2001; 10: 177-88.

22. Melo MS, Lolio CA, Lucena MAF, Kirzner CF, Martins SM, Barros MNDS. Causas múltiplas de morte em diabéticos no município de Recife, 1987. Rev Saúde Pública 1991; 25:435-42.

23. Franco LJ, Mameri C, Pagliaro H, Iopchida LC, Goldenberg P. Diabetes como causa básica ou associada de morte no Estado de São Paulo, Brasil, 1992. Rev Saúde Pública 1998; 32:237-45.

24. Currie CJ, Morgan CL, Gill L, Stott NCH, Peteers JR. Epidemiology and costs of acute hospital care for cerebrovascular disease in diabetic and nondiabetic populations. Stroke 1997; 28:1142-6.
25. Kuller LH, Velentgas P, Barzilay J, Beauchamp NJ, O' Leary DH, Savage PJ. Diabetes mellitus, subclinical cardiovascular disease and risk of incident cardiovascular disease and all-cause mortality. Arterioscler Thomb Vasc Biol 2000; 20:823-9.

26. Laurenti R, Jorge MHP, Lebrão ML, Gotlieb SLD. Estatística de saúde. São Paulo: EPU; 1987.

27. Monteiro CA, Conde WL. Evolução da obesidade nos anos 90: a trajetória da enfermidade segundo estratos sociais no Nordeste e Sudeste do Brasil. In: Monteiro CA, organizador. Velhos e novos males da saúde no Brasil: a evolução do país e suas doenças. São Paulo: Editora Hucitec/Núcleo de Pesquisas Epidemiológicas em Nutrição e Saúde, Universidade de São Paulo; 2000. p. 421-31.

28. Lotufo PA, Lolio CA. Tendências da evolução da mortalidade por doenças cardiovasculares: o caso do Estado de São Paulo. In: Monteiro CA, organizador. Velhos e novos males da saúde no Brasil: a evolução do país e suas doenças. São Paulo: Editora Hucitec/Núcleo de Pesquisas Epidemiológicas em Nutrição e Saúde, Universidade de São Paulo; 2000. p. 279-88.

Recebido em 24/Jul/2003

Versão final reapresentada em 05/Jan/2004 Aprovado em 07/Mar/2004 